\title{
Microcontroller Based Master Slave Communication for Electrical Stepper Motor
}

\author{
Rayan Mohamed Hamid ${ }^{1}$, Eltahir Mohamed Hussein ${ }^{2}$ \\ ${ }^{1}$ Department of Control, Faculty of Engineering, Al-Neelain University, Sudan \\ ${ }^{2}$ Associate Professor, Biomedical Engineering Department, Collage of Engineering, \\ Sudan University of Science and Technology (SUST), Sudan
}

\begin{abstract}
A process control systems consists of various continuous process signals coming from the various sensors and transmitters placed at different locations in the field. The signals from the sensors or transmitters may be analog or digital and this paper is a combination of analog and digital electronics. This paper is low-cost microcontroller-based on master slave communication device designed to fulfill the requirements of the industry applications. It's consists of monitoring parameters and control parameter in stepper motor then display the temperature in LCD. The PC interface is one of the main features of the project. The project is mainly consisting of the following modules: Module one is related to master circuit, module two is used to measure the temperature of the motor using a temperature sensor and module three is used to rotate the angle of stepper motor with help of microcontroller.
\end{abstract}

Keywords: Master slave communication, stepper motor, Microcontroller

\section{Introduction}

It is very much essential in laboratories as well as in industries to monitor temperature and humidity continuously. Earlier measurements had been done through manual measurements from analog instruments, such as thermometers, manometers and hygrometers. Such type of measurements can't fulfill the current Requirements in terms of the time duration and accuracy. [1,2].

The paper discuss how to monitors and controls the various parameters using the RS-232 protocol. 3 slave microcontrollers are interfaced with a Master microcontroller. And it's multi-slave single master message broadcast system that is suitable for system where data contained in short message are needed to be received at multiple locations simultaneously.

Because messages are sent to all the nodes in a system, this project is especially suited to systems where consistency in the received messages at all the receiving nodes is needed, in this case, all nodes are notified of the rejection, ensuring the data consistency across the network

Messages are sent to all nodes, but their "message identifiers" indicate whether each node should act on the message. However, all nodes participate in indicating whether the message was sent correctly, increasing the reliability of the bus.

\subsection{Objectives}

- To design and construct a "master slave communication".

- To interface hardware and software using Atmega 32 microcontroller.

- To measure and control the temperature of stepper motor.

- To display and monitor a temperature at LCD and PC.

- To develop a printed circuit board (PCB) for project circuit.

- Design a Enclosure for overall project

\subsection{Methodology}

In this research the following procedures and steps were taken:

- Building up the software.

- Running simulations programs.

- Hardware design.

- Comparison of simulation results with those obtained from experimental test.

\subsection{Block Diagram \& Description}

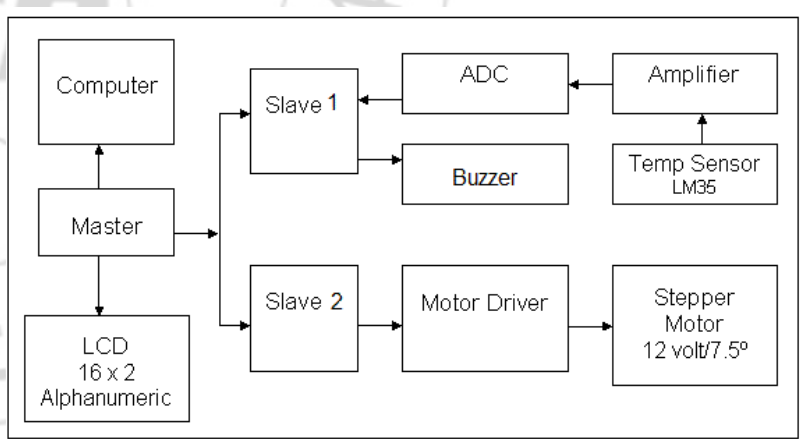

Figure 1: shows the project block diagram

\subsection{Hardware Design}

This part explains more about the hardware design and construction involves in this project. There were master slave ATMEGA32 microcontrollers, temperature sensor, a stepper motor, a Serial Communication and PCB Development and design an Enclosure for overall project.

\section{Requirements of master slave communication}

1) Microcontroller

This is the CPU (central processing unit) of our project, three microcontrollers had used. The various functions of the three microcontrollers are to measure the temperature of the 


\section{International Journal of Science and Research (IJSR) \\ ISSN (Online): 2319-7064}

Index Copernicus Value (2013): 6.14 | Impact Factor (2015): 6.391

motor using a temperature sensor and rotate the angle of stepper motor. The microcontroller used in this research was Atmega 32. Why Atmel? There are many excellent international companies that produce microcontrollers. As Atmel states, "Atmel Corporation is an industry leader in the design and manufacture of advanced semiconductors, with focus on microcontrollers, nonvolatile memory, logic, radio Frequency components and sensors." Some of the highlights of the Atmel AVR line include

- High performance coupled with low power consumption,

- Outstanding flash memory technology,

- Reduced instruction set computer Harvard Architecture,

- Single-cycle instruction execution,

- Wide variety of operating voltages (1.8--5.5 VDC),

- Architecture designed for the $\mathrm{C}$ language,

- One set of development tools for the entire AVR line, and

- In-system programming, debugging, and verification capability[3]

\section{2) Temperature Sensor ( LM35)}

The LM35 series are precision integrated-circuit temperature sensors, whose output voltage is linearly proportional to the Celsius (Centigrade) temperature. The LM35 thus has an advantage over linear temperature sensors calibrated in Kelvin [4].

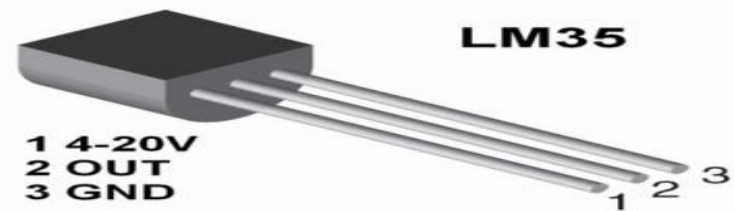

as robotics, electronic component handlers, testers, dispensers, and other manufacturing equipment [6] Stepper motor has four sets of coils. One end of each coil may be connected together and then connected to DC supply. The remaining four ends may be driven through transistors either separately or in integrated circuit form. A four-bit code sequence continuously applied to the drive circuit from the microcontroller port causes the motor shaft to rotate in angular steps [7]. The figure below show the dc stepper motor

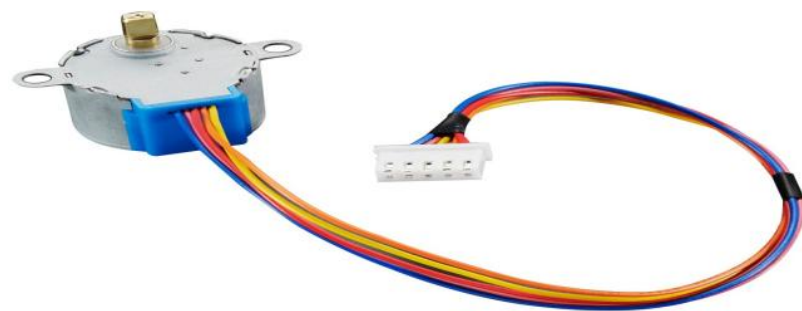

Figure 4: Dc Stepper motor [8]

\section{4) Serial Input-Output}

Serial communication (also called RS232 communication) enables a microcontroller to be connected to another microcontroller or to a PC using a serial cable. Some microcontrollers have built-in hardware called USART (universal synchronous asynchronous receiver-transmitter) to implement a serial communication interface [9].

In order communicate between Microcontroller and serial port computer; we need a connector to connect between them. Use DB9 connector for serial communication.

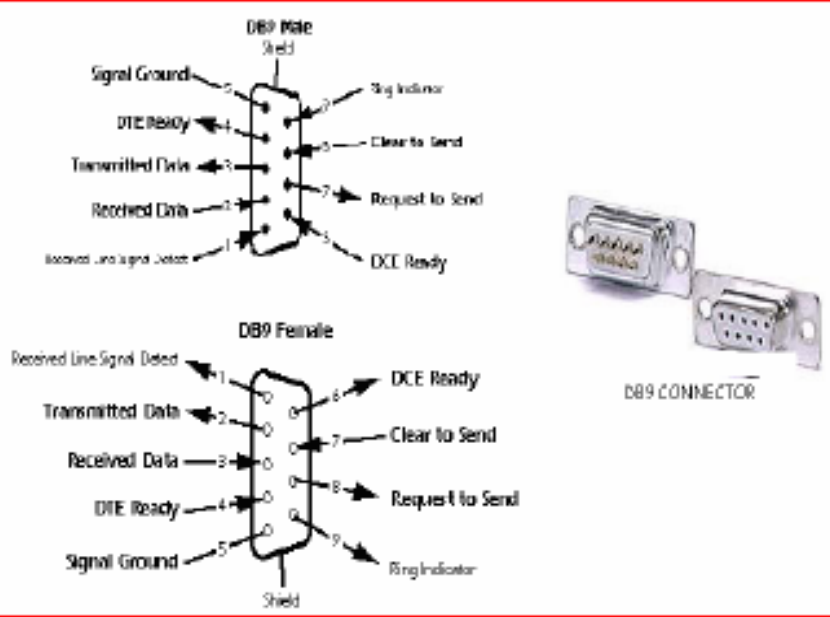

Figure 5: Serial Communication interface

\section{5) Liquid Cristal Display (LCD)}

Liquid Crystal Display (LCD) $16 \times 2$ alphanumeric have used. It can display alphabets along with numbers on 2 lines each containing 16 characters.

Figure 3: Getting data from analog world [5]

\section{3) Stepper Motor}

Stepper motors are used in a wide variety of applications. They are prevalent in consumer office. Equipment such as printers, plotters, copiers, and scanners . Stepper motors are also used in automotive applications for electronic throttle control, dashboard indicators, and climate control systems. Stepper motors are also found in industrial equipment such 

Ш山ШШШШШШ

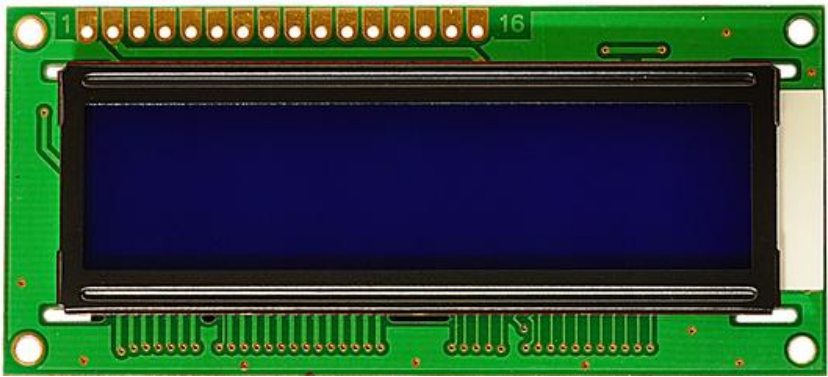

Figure 6: LCD [10]

\section{Building and Programming the Microcontroller}

The microcontroller was programmed following several steps which principally are:

- The program was been written in $\mathrm{C}$ language using BASCOM compiler

- This program is compiled so as to generate a hexadecimal file (HEX).

- The ATMEGA32 is placed into the programmer and the HEX file transferred to the microcontroller through the programmer software.

\section{Software section}

For the proper functioning of the data acquisition system first step is simulation the system by protus software

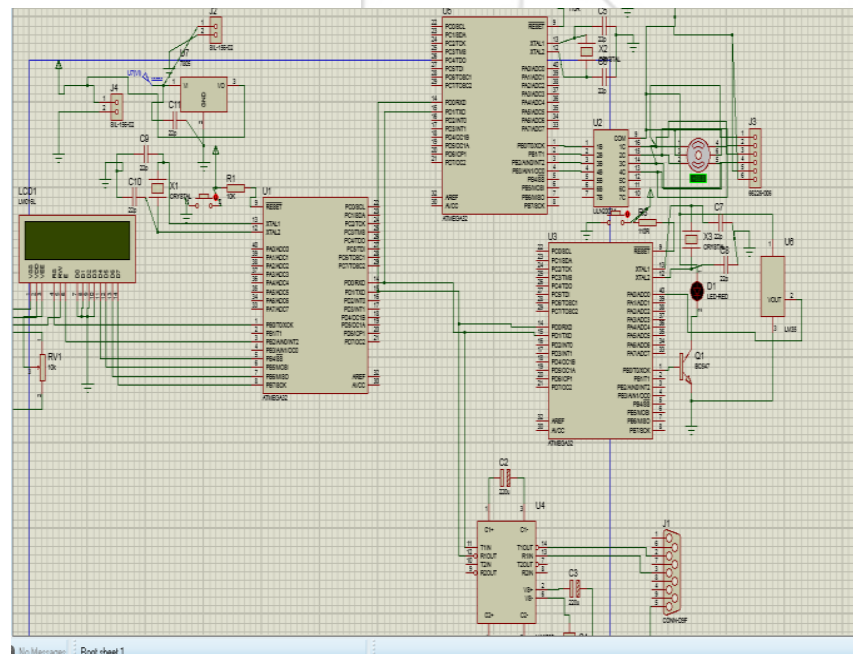

Figure 7: shows the simulation result for the whole system after the developed program Code was loaded into the microcontroller

\section{PCB Development}

This part explains about the process that has been used in order to develop a Printed Circuit Board (PCB) for project circuit board. The board is designed using a personal computer. The layout is drawn using the software "protus".

There were several step involves in this process like capture circuit using software, develop physical layout, etching, drill, insert components and circuit test. And the next figure shows the The PCB layout.

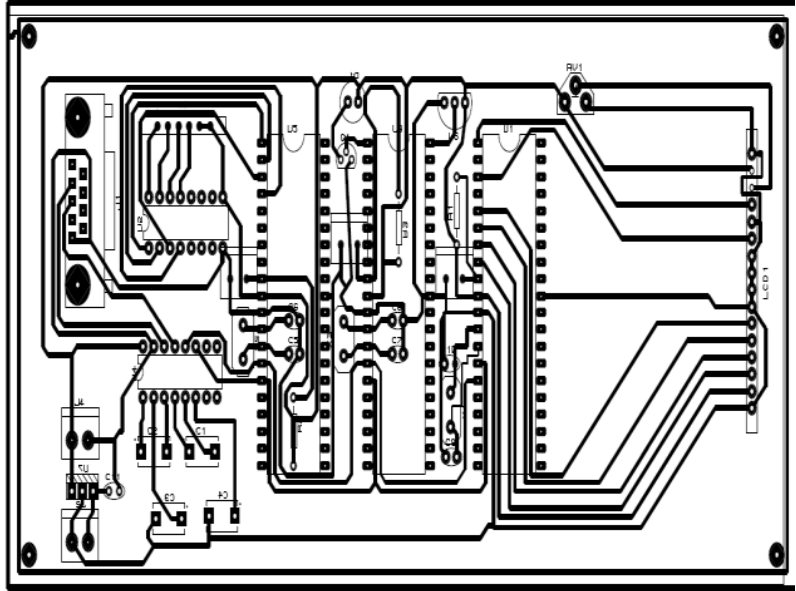

Figure 8: shows the PCB layout

\section{Application Program}

For proper acquisition of the temperature by the PC using MicroElektronika Usart Terminal

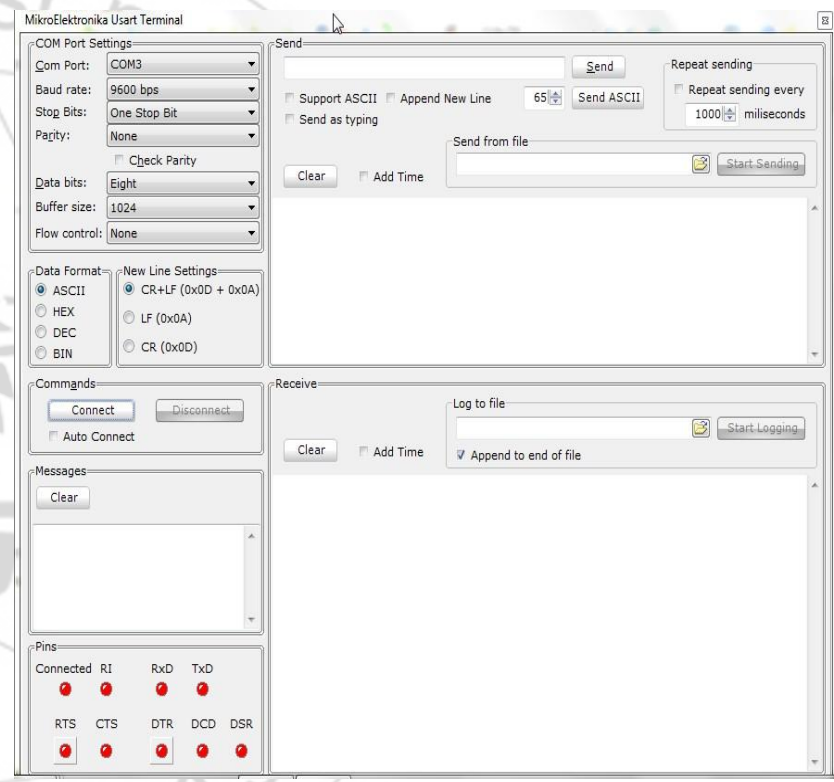

Figure 9: Shows the MicroElektronika Usart Terminal PC interface

\section{Hardware Result}

Master slave communication system has been successfully implemented and tested

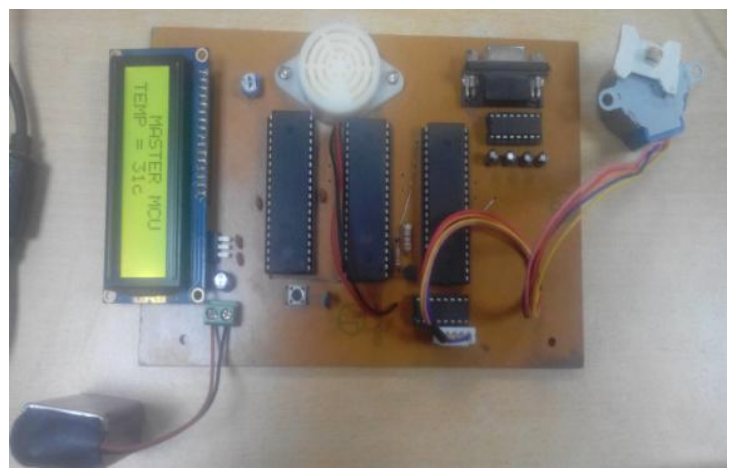

Figure 10: shows the PCB project implemented 


\section{International Journal of Science and Research (IJSR) \\ ISSN (Online): 2319-7064}

Index Copernicus Value (2013): 6.14 | Impact Factor (2015): 6.391

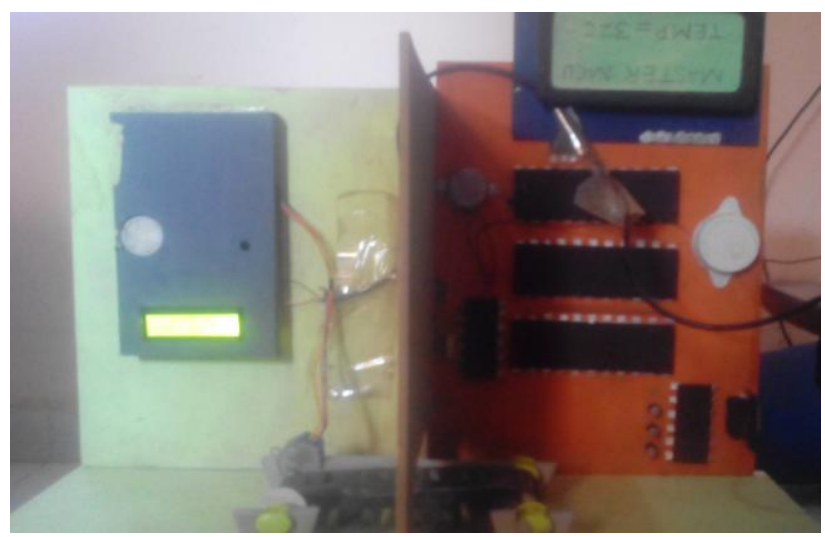

Figure 11: Shows the Enclosure project implemented

\section{Applications}

- Can be used in factory automation.

- Can be use machine control

- Medical equipment and devices.

- Textile industry

\section{Conclusion}

As a conclusion, the project was aim the objective to monitor and control (protection) the temperature of stepper motor which interface with master slave microcontroller's communication then display at PC and LCD.

\section{References}

[1] N. Monoranjan Singh, K. C. Sarma, "Low cost PC based real time data logging system using PCs parallel port for slowly varying signals", Journal of Assam Science Society, Vol 50 (1-2), pp 36-41 (Dec 2009)

[2] N. Monoranjan Singh, K. C. Sarma, "Design of PIC 12F675 microcontroller based data acquisition system for slowly varying signals", Jl. of Instrum. Soc. of India, Vol 40 (1), pp 15-17 (March 2010)

[3] Atmel AVR Microcontroller Primer: Programming and Interfacing, Daniel J. Pack Morgan \& Claypool Publishers, 2008

[4] Data sheet of LM35.

[5] THE PIC MICROCONTROLLER AND EMBEDDED SYSTEMS Using Assembly and C, Pearson Education international, 2011

[6] STEPPER MOTOR REFERENCE DESIGN, Silicon Laboratories Inc.

[7] 8051 MicrocontrollersAn Applications-Based Introduction David Calcutt ,Fred Cowan ,Hassan Parchizadeh, Linacre House, Jordan Hill, Oxford OX2 8DP , 2004

[8] www.robotshop.com access on 5/1/2016 at $4.40 \mathrm{pm}$

[9] Advanced PIC Microcontroller Projects in C, Dogan Ibrahim, Linacre House, Jordan Hill, Oxford OX2 8DP, UK. 2008

[10] www.gravitech.us Access on 29/12/2015 at 10 am 